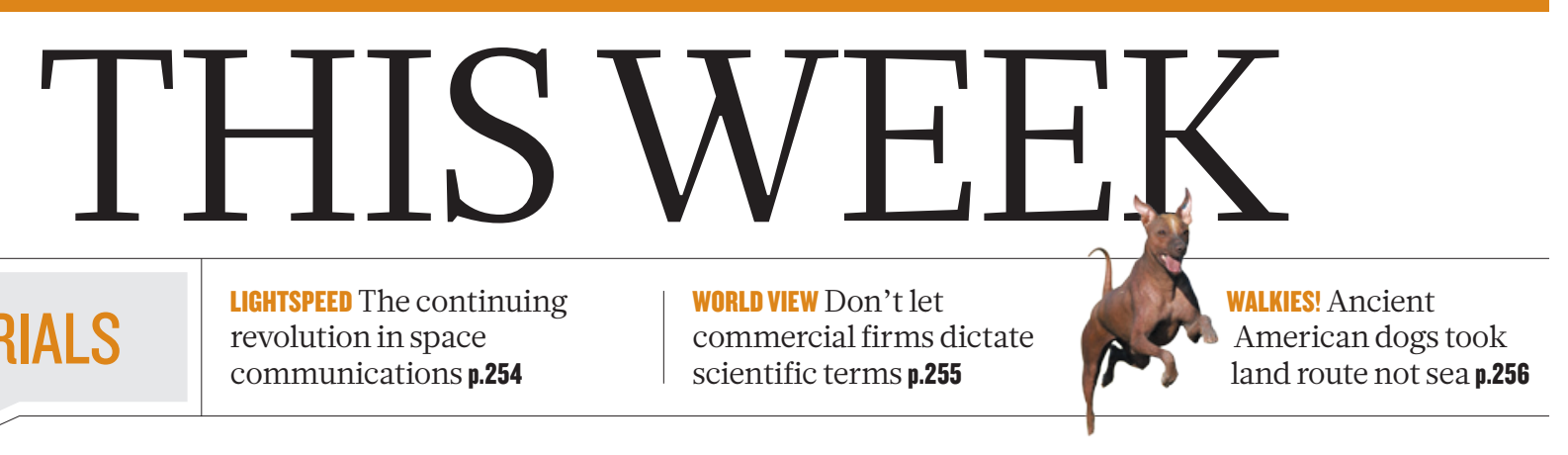

EDITORIALS revolution in space communications $\mathbf{p . 2 5 4}$ commercial firms dictate scientific terms p.255

\title{
The benefits of brain mapping
}

\author{
Two huge projects have the potential to revolutionize neuroscience, as long as they don't drain \\ money from other work and are monitored to keep them on target.
}

$\mathrm{T}$ There are roughly as many neurons in the human brain as there are pages on the Internet, give or take a million or so. But brain wiring is orders of magnitude more complex than the web. "Think about it like this," says Konrad Kording, a neuroscientist at Northwestern University in Chicago, Illinois, who is interviewed in a News Feature on page 272 that describes the task ahead for two billiondollar research programmes to understand the brain. "Whereas Internet pages only link to a couple of others in a linear way, each neuron links to thousands of others - and does so in a nonlinear way."

Neuroscientists know frighteningly little about the brain's complexity. They have sketched out the broad anatomy of the brain, and realize that individual functions - from deciding to lift a cup to feeling envy - are mediated by circuitry that crosses anatomical borders. They can examine the detailed electrical activity of small numbers of neurons. They can wield imaging technologies that show which brain areas are activated during defined tasks, such as viewing pleasant or unpleasant pictures. But those tiny (in brain terms) pieces of information have not led neuroscientists to the big picture: what we mean by human consciousness, what makes us our individual selves or why some people develop psychiatric disorders. Neuroscientists need to be able to join the dots - and there are a lot of dots.

Many scientists now believe that real progress on learning how the brain works can be made only through highly funded, interdisciplinary big science of the kind promised by US President Barack Obama's BRAIN Initiative (Brain Research through Advancing Innovative Neurotechnologies) and the European Commission's Human Brain Project, both launched this year. The first steps will be to develop technologies to map the brain in unprecedented detail, in terms of activity and anatomy - and to develop theoretical neuroscience to make sense of it.

The approach is intimidating. Each of the big programmes is expected to absorb US $\$ 1$ billion or more in the next decade (although not all of the money is yet in the bank). Some neuroscientists worry that pumping so much money into top-down programmes will reduce support for small, hypothesis-driven projects in individual labs. They are also concerned that the money could be wasted by pouring it into schemes that turn out to be unhelpful.

They are right to be nervous, and their concerns must be taken on board. The money pumped into the launch phases of the two big programmes is genuinely new - and the rest must be, too. As the programmes develop, they should not encroach on funding for research driven by individual investigators. And it is essential that the broad scientific community is involved in design and oversight of the big programmes, to ensure that the processes remain transparent, on track and based in the real world.

In some ways, the current tensions in the neuroscience community bear comparison to those surrounding the launch of the Human Genome Project in the early 1990s. New technologies had revolutionized molecular biology in the previous decade, but the outpouring of data from the project could not address the big picture of how genes fit together to keep us healthy or make us ill. The data needed to be referenced to detailed genome sequences. Many in the community bewailed the centralization and industrial scale of the work. Scientists prophesied the end of individual-investigator-driven research. But in the end, the results served to promote individual projects - and have revolutionized our understanding of many diseases. No molecular biologist now regrets the centralized investment in the Human Genome Project, even "Neuroscientists if - perhaps even because - it unexpectedly know

frighteningly

little about

the brain's

complexity."

showed that we are only partly controlled by the sequences of our DNA. Researchers are now pinning down the mechanisms through which gene expression is altered by our changing environment.

Similarly large gains are likely to emerge from the big brain projects, although they are not inevitable. The risk of failure is high, but the rewards of success will be great. In recent years, much of the pharmaceutical industry has pulled out of work on brain disorders, following many failures of candidate drugs in trials. There is an urgent need for new approaches to treating disorders that manifest in the young, such as schizophrenia or autism spectrum disorder, and those that begin in older age, such as Alzheimer's or Parkinson's disease. Reliable and detailed reference maps of the brain are likely to ground the research in reality, attracting industry back.

There is also a high-stakes cultural issue. As neuroscientists get a stronger grip on how the brain works, they will encroach ever deeper into the territory of philosophers. What does it mean to be human? The brain will contain the answer - and it won't be about surfing the Internet. -

\section{Active protection}

\section{Parents should vaccinate their children against human papillomavirus.}

S cientists at the US Centers for Disease Control and Prevention (CDC) in Atlanta, Georgia, announced good news last month. The prevalence of key strains of disease-causing human papillomavirus (HPV) fell by $56 \%$ in US girls aged $14-19$ years in the years after 2006, when a vaccine was added to the routine US immunization schedule for girls (L. E. Markowitz et al. J. Infect. Dis. 208, 385-393; 2013).

This is a clear-cut vaccine success story. The decline represents a drop from more than 1 in 10 girls in this age range carrying the 
relevant strains of the virus to just 1 in 20 . This is a significant result. About 14 million people in the United States, most in their late teens and early 20s, become infected with HPV each year. The two most dangerous strains - those targeted by vaccines made by both Merck and GlaxoSmithKline - cause cervical cancer, other anogenital cancers and throat cancer. Merck's vaccine also protects against two further strains that cause genital warts.

The vaccine is given in three doses over six months. The CDC first recommended that all girls aged 11 and 12 be vaccinated. In 2011, it said the same for boys, for whom only the Merck vaccine is licensed. The idea is to immunize children before they become sexually active. Given that HPV is the most common sexually transmitted disease, waiting longer only increases the odds that the protection will be provided too late. The vaccine has been shown to be both safe and highly effective, and continuing experience - as of March, about 56 million doses had been distributed in the United States - reinforces that.

Yet proportional uptake in the United States has been poor. In 2010, a national survey found that only $49 \%$ of girls aged 13 to 17 had received at least one dose of vaccine, and only $32 \%$ had received all three doses. By comparison, Rwanda has achieved more than $80 \%$ vaccination coverage and several Canadian provinces have reached $85 \%$.

It is possible that unvaccinated girls in the United States are already benefiting from the compliance of the parents who have stepped up to have their children immunized. When the CDC scientists explored whether the decrease in HPV prevalence among 14-19-year-old girls might be due to herd immunity, they found that vaccinated sexually active girls showed a striking $88 \%$ decrease in prevalence of the relevant HPV strains, compared with the pre-vaccine era. But they also found a $28 \%$ decrease in prevalence among unvaccinated girls. The finding was not statistically significant, and was difficult to interpret owing to differences in the reported sexual behaviour of the two groups - for instance, the unvaccinated girls reported fewer sexual partners. Nevertheless, herd immunity is a possible explanation, and other studies have indicated that it is at play.

It is worth noting that cervical cancer, almost all of which is caused by HPV, disproportionately affects black and Hispanic women in the United States, possibly because they have reduced access to screening. And among women who do contract cervical cancer, black women have proportionally the highest death rate.

But neither white people nor parents of boys of any race or ethnicity should be complacent when considering whether to vaccinate. The
"HPV is an equalopportunity infectious agent." vaccine is not only about preventing cervical cancer, nor even only about preventing anal cancer in males who have sex with males. Consider that the proportion of US throat cancers associated with HPV has exploded in recent decades among white men and women. (Similar increases have occurred in Canada and some European countries.) As the actor Michael Douglas was frank enough to acknowledge in an interview published last month, throat cancer of the type that he was treated for in 2010 is caused by HPV contracted through oral sex. (Douglas's representatives later denied that he had intended the statement to refer to his own particular case.)

The take-home message is that HPV is an equal-opportunity infectious agent. As the CDC noted when it announced the findings last month, cervical cancer is simply the most common among about 19,000 cases of cancer caused by HPV in US women each year, and throat cancer is the most frequent among 8,000 cases of such cancers in men. The costs are sobering: the CDC calculates, for instance, that 50,000 girls alive today who will get cervical cancer during their lifetimes would not have done so had the country quickly reached $80 \%$ vaccination rates.

Squeamishness among parents being asked to vaccinate 11-yearolds against a sexually transmitted disease is understandable. But in the face of such a clearly effective means of protecting our young people, ducking the issue, hoping for the best or relying on the responsible actions of others is not.

\section{Light show}

\section{Lasers will one day improve data transfer from spacecraft, but hurdles must still be overcome.}

$\mathrm{T}$ he first images from another planet trickled to Earth as slowly as an intravenous drip. On 15 July 1965, as the spacecraft Mariner 4 swept past Mars, a small television camera began to roll. It took 22 images of the Martian surface, each 200 pixels square. Mariner 4 swivelled its high-gain, 1-metre-wide parabolic radio antenna towards Earth. It began transmitting the images in the $S$ band, a high-frequency part of the radio spectrum, at a rate of 33.33 bits per second - about a million times slower than broadband Internet connections today.

Mission scientists at NASA's Jet Propulsion Laboratory in Pasadena, California, were too impatient to wait for the complete first image, and for computers to process it. So as the data arrived, the scientists pinned ticker-tape strips of the greyscale pixel readouts to a wall - each strip a line from the image - and coloured in the rugged scene by hand with pastel crayons. It would take four days for all of the images to be returned to Earth.

Interplanetary communications have since improved considerably. The $\mathrm{S}$ band gave way to the $\mathrm{X}$ band, then to $\mathrm{K}$ and $\mathrm{Ka}$ - each at a higher frequency than its predecessor, giving engineers more cycles per second to pack in information. When the Mars Reconnaissance Orbiter (MRO) went into orbit around the red planet in 2006, it was armed with antennas that could transmit information at a rate of six megabits per second. That is a jump in Mars transmission rates of more than five

orders of magnitude in the 40 years between Mariner 4 and the MRO, and a doubling of the data-transfer rate every 2 years or so. Moore's law of computing power seems to have guided planetary data transmissions just as it has the exponential rise in transistor density on a chip.

Yet scientists, ever greedy for more data, are still constricted. The $\mathrm{MRO}$, for instance, is limited in how often it can use some of its dataintensive instruments, such as the high-resolution camera. Such bottlenecks are motivating a push for optical communications (see page 266). In the coming months, both NASA and the European Space Agency will launch spacecraft with communications modules that can use lasers rather than radio transmitters. This has two benefits. First, lasers work at higher frequencies than radio does, so they can transmit more information. Second, a laser stays collimated in a narrow beam, whereas radio transmissions spread out as they travel, meaning that lower-power laser transmitters can be used to convey the same amount of information. Optical communications can also take advantage of the extraordinary investment in lasers, which have shrunk in size and grown in power.

Scientists will surely develop instruments that take advantage of the higher bandwidth. The public could benefit, too. The NASA laser demonstration, which will go to the Moon, claims to be able to return data in high definition. Imagine seeing details of the Moon broadcast live in as much detail as TV viewers spot sweat on a football player's face.

There are issues to contend with. Lasers have a hard time transmitting through clouds, and so optical communications systems may have to depend on radio relay stations orbiting Earth (or receivers

$\rightarrow$ NATURE.COM To comment online, click on Editorials at: go.nature.com/xhunqu in cloudless deserts) for the final stretch home. But it is not too difficult to envision a day when the Solar System is stocked with spacecraft all networked by pulsed light: an interplanetary Internet. 